\title{
Metformina versus la píldora anticonceptiva oral combinada para el hirsutismo, el acné y el patrón menstrual en el síndrome de ovarios poliquísticos
}

Metformin versus the combined oral contraceptive pill for hirsutism, acne, and menstrual pattern in polycystic ovary

syndrome

\section{Comentado de:}

Fraison E, et al. Cochrane Database Syst Rev. 2020 Aug 13;8(8):CD005552. PMID: $32794179^{1}$

\section{Antecedentes}

La metformina se ha propuesto como un posible tratamiento a largo plazo más seguro y efectivo que la píldora anticonceptiva oral (PAO) en mujeres con síndrome de ovarios poliquísticos (SOPQ). Es importante comparar directamente la eficacia y la seguridad de la metformina versus las PAO para el tratamiento a largo plazo de las mujeres con SOPQ. Esta es una actualización de una revisión Cochrane que compara los agentes sensibilizantes a la insulina con la PAO, y solo incluye estudios sobre la metformina.

\section{Objetivos}

Evaluar la efectividad y la seguridad de la metformina versus las PAO (solas o en combinación) para mejorar las características clínicas, hormonales y metabólicas del SOPQ.

\section{Métodos de búsqueda}

En agosto de 2019 se realizaron búsquedas en el registro de ensayos del Grupo Cochrane de Ginecología y Fertilidad (Cochrane Gynaecology and Fertility Group), en el Registro Cochrane Central de Ensayos Controlados (Cochrane Central Register of Controlled Trials, CENTRAL), en MEDLINE, Embase y CINAHL, en los registros de ensayos, se realizaron búsquedas manuales en las referencias de los artículos identificados y se estableció contacto con expertos en el campo para identificar estudios adicionales.

\section{Criterios de selección}

Se incluyeron ensayos controlados aleatorizados (ECA) sobre la administración de metformina versus PAO (solas o en combinación) para mujeres con SOPQ.

\section{Obtención y análisis de los datos}

Se utilizaron los métodos estándar recomendados por Cochrane. Los criterios de valoración principales de la revisión fueron los parámetros clínicos del hirsutismo y los eventos adversos, ya sea graves (que requirieron la interrupción del fármaco) o menores. En presencia de heterogeneidad sustancial (estadística $\mathrm{I}^{2}>50$ ), que se podría explicar por los análisis de subgrupos preespecificados sobre la base del índice de masa corporal, los subgrupos se informaron por separado.

\section{Resultados principales}

Esta es una actualización importante, en la que se identificaron 38 estudios adicionales. Se incluyeron 44 ECA (2.253 mujeres), con 39 ECA en mujeres adultas (2.047 mujeres) y cinco ECA en mujeres adolescentes (206 mujeres). La calidad de la evidencia varió de muy baja a baja. Las limitaciones principales fueron el riesgo de sesgo, la imprecisión y la inconsistencia.

\section{Metformina versus PAO}

En las mujeres adultas, no se conoce con certeza el efecto de la metformina comparada con la PAO sobre el hirsutismo en el subgrupo con un índice de masa corporal (IMC) $<25 \mathrm{~kg} / \mathrm{m}^{2}$ (diferencia de medias [DM] 0,38; intervalo de confianza [IC] del $95 \%$ : $-0,44$ a 1,19 ; tres ECA, $n=134 ; I^{2}=50 \%$, evidencia de calidad muy baja) y el IMC del subgrupo $>30 \mathrm{~kg} / \mathrm{m}^{2}$ (DM -0,38; IC del $95 \%:-1,93$ a 1,17 ; dos ECA, $n=85, I^{2}=34 \%$, evidencia de calidad baja). La metformina puede ser menos efectiva para mejorar el hirsutismo en comparación con la PAO en el subgrupo con IMC de $25 \mathrm{~kg} / \mathrm{m}^{2}$ a $30 \mathrm{~kg} / \mathrm{m}^{2}$ (DM 1,92; IC del $95 \%$ : 1,21 a 2,64; cinco ECA, $n=254, I^{2}=0 \%$, evidencia de calidad baja). La metformina puede aumentar la tasa de eventos adversos gastrointestinales graves en comparación con la PAO (odds ratio [OR] de Peto 6,42; IC del $95 \%$ : 2,98 a 13,84; 11 ECA, $n=602, I^{2}=0 \%$, evidencia de calidad baja). La metformina puede disminuir la incidencia de otros eventos adversos graves en comparación con la PAO (OR de Peto 0,20; IC del 95\%: 0,09 a 0,44; ocho ECA, $n=363$; $I^{2}$ $=0 \%$, evidencia de calidad baja). No hubo ensayos que informaran sobre eventos adversos menores. En las adolescentes, no se conoce si existen diferencias entre la metformina y la PAO en cuanto al hirsutismo y los eventos adversos.

\section{Metformina versus metformina combinada con PAO}

En las mujeres adultas, la metformina puede ser menos efectiva para mejorar el hirsutismo en comparación con la metformina combinada con la PAO (DM 1,36, IC del $95 \%$ : 0,62 a 2,11; tres ECA, $n=135, I^{2}=9 \%$, evidencia de calidad baja). No se conoce con certeza si hubo alguna diferencia entre la metformina y la metformina combinada con la PAO para los eventos adversos gastrointestinales graves (OR 0,74; IC del $95 \%$ : 0,21 a 2,53; tres ECA, $n=171, \mathrm{I}^{2}=0 \%$, evidencia de calidad baja), ni para otros eventos adversos graves (OR 0,56; IC del $95 \%$ : 0,11 a 2,82; dos ECA, $n=109, I^{2}=44 \%$, evidencia de calidad baja). No hubo ensayos que informaran sobre eventos adversos menores. En las adolescentes no hubo ensayos para esta comparación.

\section{PAO versus metformina combinada con PAO}

En las mujeres adultas, la PAO puede ser menos efectiva para mejorar el hirsutismo en comparación con la metformina combinada con la PAO (DM 0,54; IC del $95 \%$ : 0,20 a 0,89; seis ECA, $n=$ $389, I^{2}=1 \%$, evidencia de calidad baja). La PAO puede disminuir la incidencia de eventos adversos gastrointestinales graves, en comparación con la metformina combinada con la PAO (OR 0,20 IC del $95 \%$ : 0,06 a 0,72; cinco ECA, $n=228, \mathrm{I}^{2}=0 \%$, evidencia de calidad baja). No se conoce con certeza si existe alguna diferencia entre la PAO y la metformina combinada con la PAO para otros eventos adversos graves (OR 1,61; IC del $95 \%$ : 0,49 a 5,37; cuatro ECA; $n=159 ; I^{2}=12 \%$; evidencia de calidad baja). La PAO puede disminuir la incidencia de eventos adversos menores (gastrointestinales), en comparación con la metformina combinada con la PAO (OR 0,06; IC del $95 \%$ : 0,01 a 0,44; dos ECA, $n=$ $98, \mathrm{I}^{2}=0 \%$, evidencia de calidad baja). En las adolescentes no se sabe si hay diferencias entre la $\mathrm{PAO}$, en comparación con la metformina combinada con la PAO, en cuanto al hirsutismo o los eventos adversos. 


\section{Conclusiones de los autores}

En las mujeres adultas con SOPQ, la metformina puede ser menos efectiva para mejorar el hirsutismo en comparación con la PAO en el subgrupo de IMC de $25 \mathrm{~kg} / \mathrm{m}^{2}$ a $30 \mathrm{~kg} / \mathrm{m}^{2}$, pero no existe certeza acerca de si hubo diferencias entre la metformina y la PAO en los subgrupos de IMC $<25 \mathrm{~kg} / \mathrm{m}^{2}$ e IMC $>30 \mathrm{~kg} / \mathrm{m}^{2}$. En comparación con la PAO, la metformina puede aumentar la incidencia de eventos adversos gastrointestinales graves y disminuir la incidencia de otros eventos adversos graves; ningún ensayo informó sobre eventos adversos menores. La metformina sola o la PAO sola pueden ser menos efectivas para mejorar el hirsutismo, en comparación con la metformina combinada con la PAO. No se conoce con certeza si hay diferencias entre la PAO sola y la metformina sola, en comparación con la metformina combinada con la PAO, para los eventos adversos graves o menores, excepto para la PAO versus la metformina combinada con la PAO, donde la PAO puede disminuir la incidencia de eventos adversos gastrointestinales graves y menores. En las mujeres adolescentes con SOPQ, no existe certeza de que haya diferencias entre cualquiera de las comparaciones para el hirsutismo y los eventos adversos, debido a la falta de evidencia o a la calidad muy baja de la evidencia. Se necesitan más ECA grandes y bien diseñados, que estratifiquen según el IMC, para evaluar la metformina versus la PAO y las combinaciones en mujeres con SOPQ, en concreto en las adolescentes.

\section{Comentario}

El sindrome de ovario poliquístico (SOPQ) es un tema cotidiano en la práctica ginecológica. Es una de las principales causas de alteraciones del ciclo menstrual e hiperandrogenismo en mujeres en edad reproductiva ${ }^{2}$. Es importante conocer y evaluar la eficacia de diferentes opciones terapéuticas, ya que no todas las pacientes tienen los mismos deseos, objetivos ni necesidades. Es común que a algunas mujeres le molesten los ciclos menstruales espaciados o infrecuentes, mientras que a otras lo que más le incomode sea el acné y/o el hirsutismo.

Sin duda, la anticoncepción hormonal combinada (AHC) es una de nuestras grandes aliadas en el manejo del SOPQ: organiza los ciclos, otorga anticoncepción segura, en caso de que sea necesaria, y mejora los signos de hiperandrogenismo clínico: fundamentalmente el acné y, en algunos casos, también el hirsutismo.

Desde hace varios años se investiga el posible rol de la metformina para tratamiento del SOPQ. En líneas generales, lo que muestra esta revisión sistemática es que la mayoría de los estudios disponibles son imprecisos y con importantes sesgos o errores metodológicos. Sin embargo, cuando se comparó el efecto de la metformina y de la AHC sobre el hirsutismo, se observó que la combinación de ambas estrategias terapéuticas podría ser más efectiva que la AHC sola.

Debemos tener una consideración especial en la población adolescente. Si bien es verdad que la mayoría de las pacientes adultas tienen irregularidades menstruales desde la menarca, en el grupo de adolescentes es necesario tener especial cuidado en el diagnóstico y el manejo del SOPQ ${ }^{3}$. Estas pacientes, por el simple hecho de estar en una etapa de crecimiento y desarrollo, suelen tener acné, irregularidades menstruales, ovarios poliquísticos e hiperandrogenemia o hiperandrogenismo. Es importante ser prudente y tomarse varios años antes de establecer el diagnóstico de SOPQ.

\section{Conclusiones de la comentadora}

EI SOPQ es una consulta frecuente en la práctica ambulatoria. Es importante conocer las herramientas con las que contamos para su abordaje. Siempre debemos consensuar con la paciente el manejo, teniendo en cuenta sus necesidades y entendiendo que, al ser una condición crónica, éstas han de ir cambiando a lo largo de los años. Nunca debemos olvidar la importancia de la consejería en una alimentación equilibrada, un peso saludable y la práctica de ejercicio físico.

Marina Gelin [ Secciones Endocrinología Ginecológica y Climaterio y Ginecología Infantojuvenil, Hospital Italiano de Buenos Aires. marina.gelin@ hospitalitaliano.org.ar ]

Gelin M Metformina versus la píldora anticonceptiva oral combinada para el hirsutismo, el acné y el patrón menstrual en el síndrome de ovarios poliquísticos. Evid Actual Pract Ambul. 2020;23(4):e002098. Comentado de: Fraison E, et al. Metformin versus the combined oral contraceptive pill for hirsutism, acne, and menstrual pattern in polycystic ovary syndrome. Cochrane Database Syst Rev. 2020 Aug 13;8(8):CD005552. PMID: 32794179

\section{Referencias}

1. Fraison E, Kostova E, Moran LJ, et al. Metformin versus the combined oral contraceptive pill for hirsutism, acne, and menstrual pattern in polycystic ovary syndrome. Cochrane Database Syst Rev. 2020;8(8):CD005552. Available from: 0.1002/14651858.CD005552.pub3.

2. Martin KA, Anderson RR, Chang RJ, et al. Evaluation and Treatment of Hirsutism in Premenopausal Women: An Endocrine Society* Clinical Practice Guideline. J Clin Endocrinol Metab. 2018;103(4):1233-1257. Available from: 10.1210/jc.2018-00241.

3. Witchel SF, Oberfield S, Rosenfield RL, et al. The Diagnosis of Polycystic Ovary Syndrome during Adolescence. Hormone Research in Paediatrics. 2015;83(6):376-389. Available from: 10.1159/000375530. 